\title{
CONDITIONS ON A COMPACT CONNECTED LIE GROUP WHICH INSURE A "WEYL CHARACTER FORMULA"
}

\author{
JACK M. SHAPIRO
}

ABSTRACT. A theorem showing the equivalence of three conditions on a compact connected Lie group is proved. Among the corollaries is an extended "Weyl character formula" as originally stated by Bott.

In [3] Bott states a generalized "Weyl character formula" for compact connected Lie groups $G$ whose fundamental group $\pi_{1}(G)$ has no 2-torsion (recall that the original formula is stated for $G$ simply connected). As in the original character formula the critical issue surrounds the lifting of the adjoint representation $\mathrm{Ad}: G \rightarrow S O(n)$. For $\pi_{1}(G)=0$ Ad can be lifted to $\operatorname{Spin}(n)$ where $\pi: \operatorname{Spin}(n) \rightarrow S O(n)$ is the usual double cover. In the case where $\pi_{1}(G)$ has no 2-torsion, Ad can be lifted to $\operatorname{Spin}^{c}(n)$.

The purpose of this note is to prove that three properties of compact connected Lie groups are equivalent. A corollary is a proof of the assertion in $[3$, p. 178] that a character formula exists for any compact connected $G$ where $\pi_{1}(G)$ has no 2-torsion. The Theorem also lays the groundwork for the generalization in [6] of a theorem originally proved in [4] and [5] for a large number of special cases; a "Poincaré duality" result for the equivariant $K$-theory of $G / H$ where $G$ and $H$ satisfy the hypothesis of our Theorem.

The notation is borrowed from [3]. $R(G)$ is the complex representation ring of $G, R$ denotes the set of roots with $R^{+}$the subset of positive roots relative to some ordering of the Weyl chambers. If $w$ is a weight (e.g. a root) we let $e^{w}$ denote the associated element of $R(T)$ where $T$ is a maximal torus of $G . W(G)$ is the Weyl group of $G$, and we call an element $x \in$ $R(T)$ alternating, if $\phi(x)=(\operatorname{sgn} \phi) x$ for all $\phi \in W(G)$.

The adjoint representation restricted to a maximal torus $T$ provides a real representation $[g / t]$ whose complexification $[g / t] \otimes \mathbf{C}$ equals $\Sigma_{a \in R}+\left(e^{a}+e^{-a}\right)$. Finally, we let

$$
\lambda_{-1}(G / T) \equiv \sum(-1)^{p} \lambda^{p}[g / t] \otimes \mathbf{C} .
$$

Received by the editors March 11, 1974.

AMS (MOS) subject classifications (1970). Primary 20G05; Secondary 55A10.

Key words and phrases. Weyl group, roots, weights, fundamental group. 
By the multiplicative behavior of $\lambda_{t}$ (see [2, p. 118], e.g.), or directly for that matter, we see that

$$
\lambda_{-1}(G / T)=\prod_{a \in R^{+}}\left(1-e^{\alpha}\right)\left(1-e^{-\alpha}\right) .
$$

I would like to thank A. T. Vasquez for the present form of the proof. It is essentially the original done more elegantly.

Theorem. For a compact connected Lie group $G$ the following conditions are equivalent:

(1) Ad: $G \rightarrow S O(n)$ factors through $\pi: \operatorname{Spin}^{c}(n) \rightarrow S O(n)$.

(2) There exists a character $\rho: G \rightarrow S^{1}$ such that $\left(\Sigma_{a \in R}+\alpha\right)+s=2 w$. $s$ is the weight corresponding to $\rho$ restricted to $T$ and $w$ is a weight of $G$.

(3) $R(T)$ contains an alternating element $\Omega(G)$ such that $\Omega(G) \Omega(G)^{*}=$ $\lambda_{-1}(G / T)$.

Before giving the proof, which is straightforward, we comment that for semisimple G's the only character of $G$ is the trivial one, so that $s$ must be 0 . Condition (2) in that case is the more familiar one that "one-half the sum of the positive roots is a weight." In this case there is no need for $\operatorname{Spin}^{c}(n)$; we need only consider the more familiar 2-fold covering $\pi$ : Spin $(n)$ $\rightarrow S O(n)$.

Proof. For $n \geq 3$ we have an isomorphism $\pi_{1}(S O(n) \times S O(2)) \cong Z / 2 Z$ $\oplus Z$. Let $\theta: \pi_{1} \rightarrow Z / 2 Z$ be the homomorphism corresponding to mod 2 addition. By covering space theory there is a 2 -fold covering space corresponding to the subgroup $\operatorname{ker} \theta$ of $\pi_{1}$. It can be readily checked that $p: \operatorname{Spin}^{c}(n)$ $\rightarrow S O(n) \times S O(2)$ is this covering, and that $\pi: \operatorname{Spin}^{c}(n) \rightarrow S O(n)$ is the composition of $p$ and projection onto the first factor. Alternatively one can take this as a definition of $\operatorname{Spin}^{c}(n)$. Thus it follows from covering space theory that a homomorphism $\phi: G \rightarrow S O(n)$ factors through $\pi$ if and only if for some homomorphism $\rho: G-S O(2)$ the composition

$$
\pi_{1}(G) \stackrel{\pi_{1}(\phi, \rho)}{\longrightarrow} \pi_{1}(S O(n) \times S O(2)) \stackrel{\theta}{\rightarrow} \mathrm{Z} / 2 \mathrm{Z}
$$

is zero.

Suppose now that $\operatorname{Ad}=\pi \circ \tilde{\text { Ad }}$ for some homomorphism $\tilde{A d}: G \rightarrow$ $\operatorname{Spin}^{c}(n)$; then $p \circ \tilde{A d}=(A d, \rho)$ for some homomorphism $\rho: G \rightarrow S O(2)$. We may identify the integer lattice $I$ with $\pi_{1}(T)$ [1, p. 129]. We know then that the composition

$$
I \cong \pi_{1}(T) \rightarrow \pi_{1}(G) \rightarrow \pi_{1}(S O(n) \times S O(2)) \cong Z / 2 Z \oplus Z \stackrel{\theta}{\rightarrow} Z / 2 Z
$$


is zero. A moment's thought (see [1, p. 136]) shows that this homomorphism is given by $\Sigma \alpha+s$ where the $\alpha$ 's are the positive roots of $G$, and $s$ is the weight corresponding to the restriction of $\rho$ to $T$. Thus $(\Sigma \alpha+s) / 2=w$ is integer valued on $I$ and hence is a weight. This shows, when $n \geq 3$, that (1) implies (2). The argument is clearly reversible (it is necessary to recall that $\pi_{1}(T) \rightarrow \pi_{1}(G)$ is onto). If $n \leq 2$ the Theorem is trivial, for $G$ is abelian!

We now show that (2) implies (3). We will show that $\Omega(G)=e^{-w} \Pi\left(1-e^{a}\right)$ will work. Let $s_{a_{1}} \in W(G)$ denote "reflection in the wall $\alpha_{1}=0$ ". $W(G)$ is generated by such elements, thus to show $\Omega(G)$ is alternating is to show that $s_{a_{1}}(\Omega(G))=-\Omega(G)$. It is known (see [1, 5.39]) that $s_{a_{1}}\left(a_{1}\right)=-a_{1}$ and that $s_{a_{1}}$ permutes the other positive roots among themselves. Thus $s_{a_{1}}\left(\Pi\left(1-e^{a}\right)\right)=-e^{-a} 1 \Pi\left(1-e^{a}\right)$. Since $e^{s} \in R(G)=R(T)^{W(G)}$, we have $s_{a_{1}}(s)=s$. Thus $s_{a_{1}}(w)=w-a_{1}$. Hence $s_{a_{1}}(\Omega(G))=-\Omega(G)$. From the formula for $\lambda_{-1}(G / T)$ we see that $\Omega(G) \Omega(G)^{*}=\lambda_{-1}(G / T)$.

We turn now to the proof that (3) implies (2). By [1, p. 147], since $\Omega(G)$ is alternating, we can write $\Omega(G)=a \Pi\left(1-e^{a}\right)$ for some $a \in R(T)$. If $\Omega(G) \Omega(G)^{*}=\lambda_{-1}(G / T)$ we deduce that $\lambda_{-1}(G / T)=a a^{*} \lambda_{-1}(G / T) \in R(T)$. Thus $1=a a^{*}$ and $a$ is a unit. A look at the structure of $R(T) \cong$ $Z\left[x_{1}, \ldots, x_{l}, x_{1}^{-1}, \ldots, x_{l}^{-1}\right]$ shows that such an $a$ corresponds to an element of the form $\pm x_{1}^{n} \ldots x_{l}^{n}$ (i.e. $a= \pm e^{-w}$ for some weight $w$ ). Thus $e^{-w} \Pi\left(1-e^{a}\right)$ is aletrnating. Reversing one of the above calculations shows that $s_{\alpha_{1}}(w)=w-\alpha_{1}$. Hence $s=-\Sigma \alpha+2 w$ is fixed by $s_{\alpha}$. Hence it is fixed by $W(G)$. Let $e^{s}: T \rightarrow S^{1}$ be the corresponding character. We wish to show that $e^{s}$ is the restriction of a homomorphism $\rho: G \rightarrow S^{1}$. Suppose Ad factors through $\operatorname{Spin}(n)$. We may use $[1,6.19]$ and the notation introduced there. There must be an $h$ such that $A(b)=\delta$. On the other hand $A\left(b e^{s}\right)=$ $\pm \delta \rho$ for some irreducible representation $\rho$ of $G$. Since $s$ is invariant, $A\left(b e^{s}\right)=A(h) e^{s}=\delta e^{s}$. Thus $e^{s}= \pm \rho$. Viewing both as functions on $T$ and evaluating at the identity, we see that the + sign must hold (i.e. $e^{s}$ is the restriction of $\rho$ ). If Ad does not lift to Spin we may find a 2 -fold cover $f$ : $\widetilde{G} \rightarrow G$ for which Ad does lift. Applying the argument above we will find a homomorphism $\tilde{\rho}: \widetilde{G} \rightarrow S^{1}$ which is $e^{s} \circ f$ on the inverse image of $T$. In particular, $\tilde{\rho}$ is trivial on the kernel of $f$; thus $\tilde{\rho}$ is of the form $\rho \circ f$ and this $\rho$ is what we seek.

Definition. We say that $G$ satisfies condition $B$ if and only if it is a compact connected Lie group satisfying any (and hence all) of the above conditions. 
Remarks. As noted by Bott [3], if $\pi_{1}(G)$ has no 2-torsion, $G$ satisfies condition B. This is, of course, implicitly proved in the above argument. However, this is not a necessary condition. For example, $G=S O(2 n)$ works. Indeed in this case $1 / 2 \Sigma \alpha$ is a weight. Despite a remark to the contrary in [3], neither the $\pi_{1}$ condition nor condition $B$ is inherited by subgroups $H$ of maximal rank. For example, consider $G=\operatorname{Spin}(k+l)$ and $H$ the inverse image of $S O(k) \times S O(l) \hookrightarrow S O(k+l)$ for $k$ odd $\geq 3$. Finally, condition B brings the Weyl character formula into play. For, substituting $w$ for $1 / 2 \Sigma_{\alpha \in R^{+}} \alpha$ in $[1,6.6,6.16,6.18]$ does not change the proof.

\section{BIBLIOGRAPHY}

1. J. F. Adams, Lectures on Lie groups, Benjamin, New York, 1969. MR 40 \#5780.

2. M. F. Atiyah, K-theory, Benjamin, New York, 1967. MR $36 \# 7130$ 。

3. R. Bott, The index theorem for homogeneous differential operators, Differential and Combinatorial Topology (A Sympos. in Honor of Marston Morse), Princeton Univ. Press, Princeton, N. J., 1965, pp. 167-186. MR 31 \#6246.

4. J. Shapiro, $A$ duality theorem for the representation ring of a compact connected Lie group, Illinois J. Math. 18 (1974), 79-106.

5. - On the algebraic structure of the $K$-theory of $G_{2} / S U(3)$ and $F_{4} / \operatorname{Spin}(9)$, Illinois J. Math. 18 (1974), 509-515.

6. A. T. Vasquez, A Poincaré duality theorem for the equivariant K-theory of homogenous spaces (preprint).

DEPARTMENT OF MATHEMATICS, WASHINGTON UNIVERSITY, ST. LOUIS, MISSOURI 63130

INSTITUTE OF MATHEMATICS TECHNION, ISRAEL INSTITUTE OF TECHNOLOGY, HAIFA, ISRAEL 